\title{
Successful Treatment of Myelodysplastic Syndrome (MDS)- related intestinal Behçet's Disease by Up-front Cord Blood Transplantation
}

\author{
Atsushi Nonami, Katsuto Takenaka, Chinatsu Sumida, Kumiko Aizawa, Kenjiro Kamezaki, \\ Toshihiro Miyamoto, Naoki Harada, Koji Nagafuji, Takanori Teshima and Mine Harada
}

\begin{abstract}
Behçet's disease is a chronic, relapsing, inflammatory disease of unknown origin. The association of myelodysplastic syndrome and Behçet's disease is rare, and recent reports have indicated that immunosuppressive agents alone are not sufficient to control Behçet's disease associated with MDS and many patients die of infection or hemorrhage. We report a case of MDS with intestinal Behçet's disease. We performed cord blood transplantation with a myeloablative regimen as the primary treatment. The patient achieved complete remission for both diseases, which continued for more than 16 months. Our experience suggests that CBT may provide a potent therapeutic option for the treatment of MDS-related Behçet's disease.
\end{abstract}

Key words: trisomy 8, cord blood transplantation

(DOI: 10.2169/internalmedicine.46.0291)

\section{Introduction}

Myelodysplastic syndrome (MDS) is a heterogeneous group of stem-cell disorders characterized by multi-lineage dysplasia and pancytopenia due to ineffective hematopoiesis (1). Many cases show various types of cytogenetic abnormalities. Trisomy 8 is one of the most frequent abnormalities and occurs at a frequency of 8.9-20\% among MDS patients (2). Behçet's disease is a chronic, relapsing, inflammatory disease of unknown etiology characterized by recurrent oral ulcers, genital ulcers and eye and skin lesions. Intestinal lesions occur in approximately $15 \%$ of the patients; patients with diffuse intestinal ulcers are very difficult to treat and have higher mortality because of severe complications such as hemorrhage, perforation and infection (3). This disease is considered to occur due to a type of multisystem vasculitis, but the etiology is unknown. The association of these two diseases has been reported in only 28 cases (410); most of these had coexistent intestinal Behçet's disease with ulcers of the terminal ileum and without eye lesions ( 6 , 7). Interestingly, it is speculated that this is not a simple coexistence but that the aetiology of intestinal Behçet's disease is at least partly derived from MDS itself $(2,5,11,12)$. Furthermore, most of these patients (21 of 28) had trisomy 8 chromosome abnormality. Kimura et al (9) investigated the relationship between trisomy 8 and intestinal Behçet's disease in patients with MDS and reported the relationship to be statistically significant. Moreover, they discussed the risk factors in MDS for coexistence with intestinal Behçet's disease to be Japanese origin, young age, comparatively few blasts and the presence of trisomy 8 .

Recent reports have indicated that the control of intestinal Behçet's disease associated with MDS using immunosuppressive agents, such as prednisolone, alone is difficult and many patients die of infection or hemorrhage $(4,6,9)$. On the other hand, the efficacy of allogeneic haematopoietic stem-cell transplantation (HSCT) for MDS has been established.

Here, we report a case of MDS-related intestinal Behçet's disease, which was successfully treated by up-front cord blood transplantation (CBT) with a myeloablative regimen from an unrelated donor. 


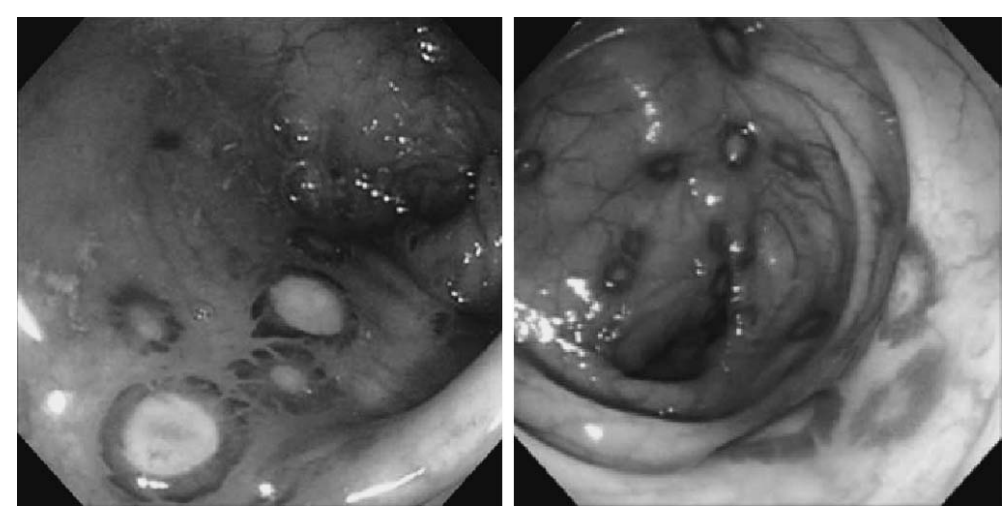

Figure 1. Total colonoscopy was performed; diffuse mucosal edema of the entire colon was revealed in addition to multiple bleeding ulcers with an erythematous and elevated margin throughout the colon.

Table 1. Laboratory Data on Admission

\begin{tabular}{lll} 
WBC & $1.0 \times 10^{9}$ & \multicolumn{1}{c}{} \\
Neut & 26.5 & $\%$ \\
Lym & 36.0 & $\%$ \\
Mono & 31.5 & $\%$ \\
Eos & 3.0 & $\%$ \\
Blast & 4.0 & $\%$ \\
RBC & $281 \times 10^{2}$ & \multicolumn{1}{l}{} \\
Hb & 7.9 & $\mathrm{~g} / \mathrm{dl}$ \\
MCV & 92.2 & $\%$ \\
MCH & 28.1 & $\mathrm{pg} / \mathrm{ml}$ \\
MCHC & 30.5 & $\%$ \\
Plt & $3.9 \times 10^{9}$ & $\mathrm{~L}$ \\
Ret & 1.8 & $\%$ \\
APTT- T/C & $53.3 / 34.5$ & $\mathrm{sec}$ \\
Fib & 337 & $\mathrm{mg} / \mathrm{dL}$ \\
FDP & 2.6 & $\mu \mathrm{g} / \mathrm{dL}$
\end{tabular}

\section{Case Report}

A 28-year-old woman was admitted in August 2005 with complaints of fever and abdominal pain. At the age of 10 , she was diagnosed with anemia. After extensive clinical and laboratory examinations, she was diagnosed as aplastic anaemia. Her complete blood cell count (CBC) revealed the leukocyte count as $1.5 \times 10^{9}$ per litre, hemoglobin concentration as $11 \mathrm{~g} / \mathrm{dL}$ and platelet count as $70 \times 10^{9}$ per litre. She was carefully followed at our outpatient clinic without medication. She had recurrent oral aphthae in the past 2 years. In April 2004, her chromosomal analysis of bone marrow showed an abnormal karyotype: 46, XX, dup (1) in 11 out of 20 metaphases analyzed. In May 2005, she complained of fever and severe abdominal pain. In July, CBC revealed a relative decrease in the leukocyte count $\left(2.6 \times 10^{9}\right.$ per litre $)$ with $23 \%$ neutrophils and $2 \%$ myeloblasts, and a considerable decrease in hemoglobin concentration $(8.5 \mathrm{~g} / \mathrm{dL})$ and platelet count $\left(28 \times 10^{9}\right.$ per litre); she was admitted to a regional hospital. Bone marrow examination showed hypocellularity with $4 \%$ blasts. The presence of trisomy 8 was detected in 18 out of 20 metaphases analyzed. On the basis of these findings, the patient was diagnosed with refractory anemia with excess blasts (RAEB) of MDS. For the workup of moderately severe anemia and abdominal pain, total

$\begin{array}{lll}\text { TP } & 6.6 & \mathrm{~g} / \mathrm{dL} \\ \text { Alb } & 3.8 & \mathrm{~g} / \mathrm{dL} \\ \text { BUN } & 12 & \mathrm{mg} / \mathrm{dL} \\ \text { Cr } & 0.53 \mathrm{mg} / \mathrm{dL} \\ \text { TB } & 0.8 \mathrm{mg} / \mathrm{dL} \\ \text { AST } & 157 \mathrm{U} / \mathrm{L} \\ \text { ALT } & 196 \mathrm{U} / \mathrm{L} \\ \text { LDH } & 316 \mathrm{U} / \mathrm{L} \\ \text { CRP } & 6.54 \mathrm{mg} / \mathrm{dL} \\ & & \\ & & \\ \text { Culture of stool } & \\ \text { Clostridium difficile (CD) toxin } & (-) \\ \text { anti-Shigella antibody } & (-) \\ \text { EBV, CMV-PCR } & (-) \\ \text { endotxoin, } \beta \text {-D glucan } & (-)\end{array}$

colonoscopy was performed. Diffuse mucosal oedema of the entire colon was revealed in addition to multiple bleeding ulcers with an erythematous and elevated margin throughout the colon (Fig. 1). For the treatment of febrile neutropenia, she received an antibiotic therapy with granulocytestimulating factor (G-CSF) administration. However, because of increasing blasts, the administration of G-CSF was discontinued, and liver dysfunction probably due to druginduced hepatitis developed. The leukocyte count gradually decreased to $1 \times 10^{9}$ per litre with $5 \%$ blasts in peripheral blood and $10 \%$ in bone marrow in August. For further evaluation of the intestinal lesions and therapeutic options including stem-cell transplantation, she was referred to us at the end of August.

Physical examination revealed that she was anaemic and had diffuse and severe tenderness in the lower abdomen. Oral or genital aphthae and erythema nodosum were not found. Uveitis was not observed either. She did not have any neurological abnormalities. Laboratory data are shown in Table 1. Abdominal computed tomography (CT) scans showed marked oedema of the ileocecum region and inflammation of the surrounding fat tissue (Fig. 2). Bone marrow was hypocellular with increased myeloblasts (13\%) and micromegakaryocytes. Chromosome analysis revealed additional several types of abnormalities as follows: 1) 46, XX, dup (1) (q42q21) (4 of 20 metaphases analysed); 2) 47, XX, 


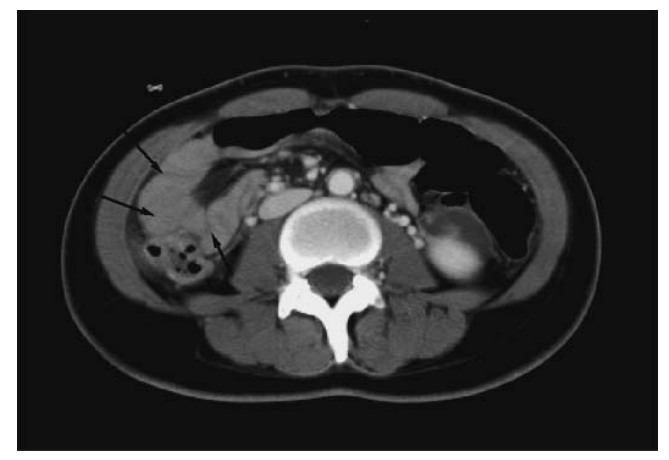

Figure 2. CT scans showed marked edema of the ileocecum region (arrows) and inflammation of the surrounding fat tissue.

dup (1) (q42q21), +8 (12/20); 3) 47, X, t (X;12) (p11.2; p 13), dup (1) (q42q21), +8 (1/20); 4) 46, XX (3/20). According to the International Prognostic Scoring System (IPSS), the disease was classified as Intermediate-2. Considering the rapid progression of the disease, we employed allogeneic HSCT as a primary therapeutic option. There was no appropriate human leukocyte antigen (HLA)-identical sibling. The disease status did not allow us to search for a volunteer donor of bone marrow through the Japan Marrow Donor Program because the search requires several months. Thus, we decided to perform CBT with myeloablative regimen. Written informed consent for this therapy was obtained.

Next, to evaluate the persistent severe abdominal pain, we performed colonoscopy. Multiple ulcers with red halo throughout the colon and erosion with depression at the terminal ileum were observed. Sections of the biopsy specimen showed moderate chronic inflammation with inflamed granulation tissue in the ulcers. Epithelioid cell granuloma, inclusion bodies of cytomegalovirus (CMV) infection, amoeba and infiltrating blasts were not observed. From these findings, invasion of blasts or viral infection was unlikely as a cause of multiple ulcers. It has been reported that MDS patients complicated with intestinal Behçet's disease often have trisomy 8 (7). Thus, along with recurrent oral aphthae in the past two years, multiple ulcers in this patient were diagnosed as intestinal Behçet's disease. We did not use prednisolone or other immunosuppressive drugs for the treatment of ulcers; instead, we started total parenteral nutrition. Because coagulation tests showed a prolonged activated partial thromboplastin time (APTT) and elevated fibrin degradation products (FDP), dalteparin was used to prevent thrombosis, which has been reported to be increased in MDS patients with trisomy 8 associated with Behçet's disease (9). Barium enema performed after 2 weeks showed little improvement of the ulcers, but there was a longitudinal ulcer at the terminal ileum. The leukocyte count dropped to $0.66 \times 10^{9}$ per litre with $15 \%$ neutrophils, but G-CSF was not used because it may act as an exacerbating factor for ulcers and thrombosis in MDS patients with trisomy 8 associated with Behçet's disease (9).

In early October, she underwent CBT from an unrelated man donor through the Japan Cord Blood Bank Network. The donor had one locus-mismatch at HLA-DR: the patient's HLA phenotype was A26, A33, B39, B44, DR15 and DR8, and that of the donor was A26, A33, B39, B44, DR13 and DR8. The number of cord blood cells infused was $2.88 \times 10^{7}$ per kilogram. The pretransplant conditioning regimen consisted of cyclophosphamide $(60 \mathrm{mg} / \mathrm{kg})$ for 2 days and 12 Gy total body radiation with six fractionations. Methotrexate $\left(10 \mathrm{mg} / \mathrm{m}^{2}\right.$ on day 1 and $7 \mathrm{mg} / \mathrm{m}^{2}$ on days 3 and 6) and cyclosporin A (3 mg/kg) were used for graftversus-host disease (GVHD) prophylaxis. G-CSF $(5 \mu \mathrm{g} / \mathrm{kg}$ per day) was administered from day 3 to facilitate myeloid recovery. Neutropenic fever occurred frequently but no apparent infection was documented. Skin GVHD (stage 1) developed on day 14 , but it gradually subsided spontaneously. Neutrophil engraftment (more than $0.5 \times 10^{9}$ per litre) was documented on day 25 and complete chimerism was confirmed by using short tandem repeat (STR). Platelet engraftment (more than $50 \times 10^{9}$ per litre without transfusion) was observed on day 49. Colonoscopy performed on day 38 showed only multiple ulcer scars without active ulcers. On day 43, CMV antigenemia $(2 / 76,600)$ was detected; it was controlled with ganciclovir and disappeared on day 62. The patient was discharged on day 101, and is now doing well in complete remission of MDS without chronic GVHD and abdominal symptoms at the time of reporting (day 480).

\section{Discussion}

The efficacy and feasibility of autologous HSCT for hematological malignancies such as non-Hodgkin lymphoma and multiple myeloma have been established. Recently, it is also used as a new therapy for the treatment of severe autoimmune diseases such as multiple sclerosis, systemic sclerosis and systemic lupus erythematosus (13-15). Autologous HSCT has also been performed on three patients with Behçet's disease (16-18). These cases suggest the possible feasibility and efficacy of autologous HSCT for the treatment of severe Behçet's disease.

Recent reports have suggested that administration of immunosuppressive agents alone is not sufficient to control MDS-related intestinal Behçet's disease, and many patients have died of infection (6), hemorrhage (4) or recurrence of ulcers (9) before the treatment of MDS. One reason for the difficulty in controlling MDS-related Behçet's disease is that underlying causes of immune abnormalities are at least partly derived from MDS itself $(2,5,11,12)$. This means that MDS should be treated concurrently. If Behçet's disease is severe, it is considered that allogeneic HSCT is necessary for the control of Behçet's disease through not only immunoablation but also the eradication of abnormal MDS clones, especially when MDS is advanced or progressive. In a previous report, the patient was treated first with steroids, wherein the endoscopic findings dramatically improved but the reduction of steroids again worsened the intestinal ulcers. The patient received CBT with myeloablative regimen, 
after which both MDS and Behçet's disease went into remission (7). Furthermore, Tomonari et al (8) reported a patient with Behçet's disease whose symptoms were refractory to conventional therapies with nonsteroidal antiinflammatory drugs, colchicine, and prednisolone, achieving complete remission after undergoing CBT with myeloablative regimen. Thus, these reports suggest the feasibility and efficacy of allogeneic HSCT with myeloablative regimen for the treatment of MDS-related severe Behçet's disease. On the other hand, Handa et al reported a case of MDS-related Behçet's disease in which symptoms of Behçet's disease exacerbated and the patient died of pulmonary haemorrhage probably due to Behçet's disease before HSCT (4). In the present case, we performed CBT for our patient as an initial therapy, and the patient achieved complete remission of both diseases. Considering these cases and the present case, we propose that chemotherapy or HSCT, rather than conventional immunosuppressive therapy, should be performed immediately to control intestinal disease as a primary therapy for MDS-related severe Behçet's disease, especially when MDS is advanced or progressive.
In conclusion, we performed CBT with myeloablative regimen as a primary therapy from an unrelated donor for treatment of MDS and MDS-related intestinal Behçet's disease. The patient achieved complete remission. This is the first case successfully treated by up-front allogeneic HSCT. Considering the past cases $(7,8)$ and our case, allogeneic myeloablative HSCT including CBT without delay may be one of the therapeutic options for the treatment of MDSrelated severe Behçet's disease, although the efficacy of bone marrow transplantation or peripheral blood stem cell transplantation has not been shown. More patients and longer follow-up are necessary to assess the efficacy of this modality in the treatment of high-grade MDS-related severe Behçet's disease. The indication of allogeneic HSCT for low-grade MDS-related severe Behçet's disease or Behçet's disease per se should also be assessed.

\section{Acknowledgement}

This work was partly supported by a research fund "Research Group on Advanced Immunosuppressive Strategies for Autoimmune Diseases (H17-immunology-011)".

\section{References}

1. Greenberg PL. The smoldering myeloid leukemic states: clinical and biologic features. Blood 61: 1035-1044, 1983.

2. Ohno E, Ohtsuka E, Watanabe K, et al. Behçet's disease associated with myelodysplastic syndromes. A case report and a review of the literature. Cancer 79: 262-268, 1997.

3. International Study Group for Behçet's Disease. Criteria for diagnosis of Behçet's disease. Lancet 335: 1078-1080, 1990.

4. Handa T, Arai Y, Mitani K. Myelodysplastic syndrome associated with intestinal tract-type Behçet disease characterized by an esophageal ulcer. J Clin Hematol 45: 135-137, 2004 (in Japanese).

5. Ogawa $H$, Kuroda $T$, Inada $M$, et al. Intestinal Behçet's disease associated with myelodysplastic syndrome with chromosomal trisomy 8 - a report of two cases and a review of the literature. Hepatogastroenterology 48: 416-420, 2001.

6. Tsubata R, Suzuki F, Sugihara T, et al. An autopsy case of intestinal Behçet's disease with sacroiliitis accompanied by myelodysplastic syndrome with trisomy 8. Jpn J Clin Immunol 28: 48-55, 2005 (in Japanese).

7. Yamato K. Successful cord blood stem cell transplantation for myelodysplastic syndrome with Behçet disease. Int J Hematol 77: 82-85, 2003.

8. Tomonari A, Tojo A, Takahashi T, et al. Resolution of Behçet's disease after HLA-mismatched unrelated cord blood transplantation for myelodysplastic syndrome. Ann Hematol 83: 464-466, 2004.

9. Kimura S, Kuroda J, Akaogi T, Hayashi H, Kobayashi Y, Kondo M. Trisomy 8 involved in myelodysplastic syndromes as a risk factor for intestinal ulcers and thrombosis-Behçet's syndrome. Leuk Lymphoma 42: 115-121, 2001.

10. Adachi $Y$, Tsutsumi A, Murata $\mathrm{H}$, et al. Behçet's disease accompanied by myelodysplastic syndrome with trisomy 8: two case reports and a review of 15 Japanese cases. Mod Rheumatol 13: 90-

\section{3, 2003.}

11. Niwa Y, Miyake S, Sakane T, Shingu M, Yokoyama M. Autooxidative damage in Behçet's disease-endothelial cell damage following the elevated oxygen radicals generated by stimulated neutrophils. Clin Exp Immunol 49: 247-255, 1982.

12. Yano K, Eguchi K, Migita K, et al. Behçet's disease complicated with myelodysplastic syndrome: a report of two cases and review of the literature. Clin Rheumatol 15: 91-93, 1996.

13. Binks M, Passweg JR, Furst D, et al. Phase I/II trial of autologous stem cell transplantation in systemic sclerosis: procedure related mortality and impact on skin disease. Ann Rheum Dis 60: 577584, 2001.

14. Hough RE, Snowden JA, Wulffraat NM. Haemopoietic stem cell transplantation in autoimmune diseases: a European perspective. Br J Haematol 128: 432-459, 2005.

15. Griffith LM, Pavletic SZ, Tyndall A, et al. Feasibility of allogenic hematopoietic stem cell transplantation for autoimmune disease: position statement from a National Institute of Allergy and Infectious Diseases and National Cancer Institute-Sponsored International Workshop, Bethesda, MD, March 12 and 13, 2005. Biol Blood Marrow Transplant 11: 862-870, 2005.

16. Hensel M, Breitbart A, Ho AD. Autologous hematopoietic stemcell transplantation for Behçet's disease with pulmonary involvement. N Engl J Med 344: 69, 2001.

17. Maurer B, Hensel M, Max R, Fiehn C, Ho AD, Lorenz HM. Autologous haematopoietic stem cell transplantation for Behçet's disease with pulmonary involvement: analysis after 5 years of follow up. Ann Rheum Dis 65: 127-129, 2006.

18. Rossi G, Moretta A, Locatelli F. Autologous hematopoietic stem cell transplantation for severe/refractory intestinal Behçet disease. Blood 103: 748-750, 2004.

(C) 2007 The Japanese Society of Internal Medicine http://www.naika.or.jp/imindex.html 